\title{
High-field fluctuation magnetoconductivity and Hall reversal response in the $\mathrm{Hg}(\mathrm{Re}) \mathrm{Ba}_{2} \mathrm{Ca}_{2} \mathrm{Cu}_{3} \mathrm{O}_{8+\delta}$ superconductor
}

\author{
Jairo Roa-Rojas*, Jorge Andrés Cardona-Vásquez, David Landínez-Téllez
}

Grupo de Física de Nuevos Materiales, Departamento de Física, Universidad Nacional de Colombia, Bogotá, Colombia

\begin{abstract}
Systematic experiments of high-field (up to $50 \mathrm{kOe}$ ) fluctuation magnetoconductivity and Hall magnetoresistivity in $\mathrm{Hg}_{1-\mathrm{x}} \mathrm{Re}_{\mathrm{x}} \mathrm{Ba}_{2} \mathrm{CaCu}_{3} \mathrm{O}_{8+\delta}(x=0.18)$ polycrystalline samples growth by means the quartz tube technique are reported. The analysis of the experimental data was performed by using the recognized Kouvel-Fisher method, which is frequently applied to study of critical phenomena. Very close to the critical temperature $T_{C}$, a genuinely critical regime of fluctuations characterized by the critical exponent $\lambda_{c}=0.32 \pm 0.01$ was identified in absence of magnetic fields. This result is consistent with the full dynamic 3D-XY universality class predicted by the model $\mathrm{E}$ of Hohenberg-Halperin with a dynamic critical exponent $z=3 / 2$. The genuine critical regime become be unstable on the application of external magnetic fields $H \approx 0.1 \mathrm{kOe}$. Near above the critical temperature $T_{C}$, the determined exponent $\lambda_{G 3}=0.52 \pm 0.02$ was interpreted as corresponding to homogeneous fluctuations, which develop in a space with three-dimensional geometry. This region is destroyed upon the application of magnetic fields above $0.5 \mathrm{kOe}$. Increasing the temperature, evidences of a homogeneous two-dimensional behavior are observed by means the identification of a $\lambda_{G 2}=1.02 \pm 0.04$. Applied fields $H>20 \mathrm{kOe}$ destroy this fluctuation regime. Far above $T_{C}$, effects of disorder and planar anisotropy produce a fluctuation spectrum characterized by a fractal topology with a critical exponent $\lambda_{G 2-G I}=1.32 \pm 0.04$. At last, very far $T_{C}$, a temperature region with $\lambda_{G I}=1.52 \pm 0.04$ was experimentally identified. This corresponds to the confinement of the quasi-particles into the Lowest-Landau-Level, due to the quantization of the electronic states around the axe of application of the external field. Measurements of Hall were performed. In the normal phase, the Hall resistivity is hole-like and inversely proportional to the temperature. In the mixed phase and when the applied field is below $\mu_{0} H=2 T$, the Hall resistivity shows a double sign reversal. For fields above $2 T$, the Hall resistivity remains positive, although qualitatively showing the trends observed at low fields. We attribute this behavior to two independent contributions with opposite sign. A negative term due to thermal fluctuations is relevant near $T_{C}$, whereas a positive contribution related to vortex motion dominates at lower temperatures. Near the zero resistance state, the Hall resistivity varies as a power law of the longitudinal resistivity, with a field independent exponent $\beta=1.41$.
\end{abstract}

PACS: 74.40.+k; 74.25.Bt; 74.60.Ec; 74.72.Bk

Key words: Fluctuation conductivity, Critical phenomena, Mixed state, High-temperature superconductivity

Fluctuaciones en la magneto conductividad de alto campo y respuesta Hall inversa en el superconductor $\mathrm{Hg}(\mathrm{Re}) \mathrm{Ba}_{2} \mathrm{Ca}_{2} \mathrm{Cu}_{3} \mathrm{O}_{8+\delta}$

\section{Resumen}

En el presente trabajo reportamos experimentos sistemáticos de fluctuaciones en la magnetoconductividad bajo la aplicación de altos campos magnéticos (hasta $50 \mathrm{kOe}$ ) y respuesta Hall en muestras policristalinas de $\mathrm{Hg}_{1-}$ ${ }_{x} \mathrm{Re}_{\mathrm{x}} \mathrm{Ba}_{2} \mathrm{CaCu}_{3} \mathrm{O}_{8+\delta}(x=0.18)$ crecidas mediante la técnica del tubo de cuarzo. Los análisis experimentales fueron realizados a través del método de Kouvel-Fisher, el cual es frecuente utilizado en estudios de fenómenos críticos. Muy cerca de la temperatura crítica $T_{C}$ y en ausencia de campo magnético fue identificado un régimen de fluctuaciones genuinamente críticas caracterizado por el exponente $\lambda_{c}=0.32 \pm 0.01$. Este resultado es consistente con el modelo 3D-XY cuya universalidad dinámica es predicha por el modelo E of Hohenberg-Halperin con un exponente crítico dinámico $z=3 / 2$. Este régimen se torna inestable bajo la aplicación de campos magnéticos superiores a $H \approx 0.1$ $k O e$. Cerca y arriba de $T_{C}$, se observe un exponente $\lambda_{G 3}=0.52 \pm 0.02$ que fue interpretado como correspondiente a fluctuaciones homogéneas desarrollándose en un espacio de geometría tridimensional. Esta región fue destruida cuando campos magnéticos superiores a $H=0.5 \mathrm{kOe}$ fueron aplicados. Al aumentar la temperatura, se evidenció un comportamiento de fluctuaciones homogéneas bidimensionales identificadas mediante el exponente $\lambda_{G 2}=1.02 \pm 0.04$. Este régimen desapareció al aplicar campos magnéticos $H>20 \mathrm{kOe}$. Lejos y arriba de $T_{C}, \operatorname{los}$ efectos de desorden de anisotropía planar produjeron un espectro de fluctuaciones caracterizados por una topología fractal con un exponente 
crítico $\lambda_{G 2-G I}=1.32 \pm 0.04$. Muy lejos en temperatura y arriba de $T_{C}$, se identificó un régimen de fluctuaciones con exponente $\lambda_{G I}=1.52 \pm 0.04$, el cual fue interpretado como relativo al confinamiento de cuasipartículas en el nivel más bajo de Landau, debido a la cuantización de estados electrónicos alrededor del eje de aplicación del campo magnético externo. Por otro lado, se efectuaron medidas de respuesta Hall. En la fase normal, la resistividad Hall fue de tipo hueco e inversamente proporcional a la temperatura. En el estado mixto y bajo la aplicación de un campo magnético inferior a $20 \mathrm{kOe}$ la resistividad Hall mostró una doble inversión de signo. Para campos por encima de este valor, la resistividad Hall permaneció positiva pero conservando la misma forma cualitativa observada a bajos campos. Este comportamiento fue atribuido a la existencia de dos contribuciones independientes de signo opuesto: ana negativa debida a fluctuaciones térmicas cerca de $T_{C}$, y otra positiva debida a movimiento de vórtices que domina a menores temperaturas. Cerca al estado en que la resistividad se anula, la respuesta Hall varía en forma de una función potencial de la respuesta longitudinal, con un exponente independiente del campo aplicado $\beta=1.41$.

PACS: $74.40 .+\mathrm{k} ;$ 74.25.Bt; 74.60.Ec; 74.72.Bk

Palabras clave: fluctuaciones en la conductividad, fenómenos críticos, estado mixo, superconductividad de alta temperatura

\section{Introduction}

It is known that in the phase transitions of second order the thermodynamic fluctuations of the order parameter play an important role in their description (Stanley, H.E., 1971). High temperature superconductors (HTSC) exhibit characteristics, which are very different from those properties of conventional low temperature superconductors. In first, the superconducting transition is enhanced and nonequilibrium Cooper pairs can be to occur in temperature intervals above the critical temperature $T_{C}$. These are the origin of precursor effects of the superconducting phase still in the normal state. Some equilibrium and transport properties change considerably in the neighborhood of the transition due to contribution of these fluctuation states.

Ginzburg (1960) effectuated the first estimation of the fluctuation effects in the specific heat of superconductor materials near $T_{C}$ (Varlamov \& Ausloos, 1997). Based on Ginzburg-Landau theory, it was shown that the superconducting fluctuations increase the specific heat above and very close to $T_{C}$. In 1968 was formulated the first microscopic theory for electric conductivity fluctuations of superconductors in the proximities of $T_{C}$, which are known as Aslamazov-Larkin theory (1968). These studies shown that the size of the fluctuation effects vary inversely proportional to the coherence length $\mathrm{x}$, which determines the spatial response of superconductor. HTSC possess an electronic excitation spectrum extremely anisotropic and very short coherence length. As a result of these characteristics, the temperature region dominated by thermal fluctuations may be attaining some ten degrees (Lobb, C.J., 1987).

\footnotetext{
*Correspondencia:

Jairo Roa-Rojas, jroar@unal.edu.co

Recibido: 31 de marzo de 2014

Aceptado: 19 de mayo de 2014
}

The manifestation of superconducting fluctuations above $T_{C}$ is conveniently demonstrated in the electric conductivity case. In first approximation, this is reduced to four distinctive effects:

i) First effect is direct and consists in the apparition of nonequilibrium Cooper pairs, with characteristic fluctuation time $\tau_{G L} \sim \frac{\hbar}{T-T_{c}}$ very close to $T_{C^{\circ}}$. A number of these pairs (depending of the proximity to $T_{C}$ ) is ever present in certain unitary volume of the normal phase. Concerning to electric conductivity, we could be to say that in $T>$ $T_{C}$, a new transference channel of charge, non-dissipative, is opened as a consequence of presence of metastable Cooper pairs. This direct effect of fluctuations on the conductivity is known as paraconductivity or AslamazovLarkin contribution.

ii) Another consequence of the formation of vanishing-Cooper pairs is the decreasing of the electronic state densities into the Fermi level. When some electrons involve in the pairing, they can not to participate simultaneously in the charge transference and in the specific heat as excitations of single particle. The several numbers of electronic states can be to change due to Cooper interaction and only could be occurs one distribution of levels along to the energetic axe. Then, one pseudo-gap of fluctuations in the Fermi level is opened (Di Castro, Castellani, Raimondi \& Varlamov, 1990). The decreasing of state densities of single electron into de Fermi level arise a reduction of the electric conductivity in the normal state. This indirect correction to fluctuation contribution on the electric conductivity is denominated contribution of the state densities. This have opposite sign when the temperature approximates to $T_{C^{+}}$from the normal state and may be singularly small when compared with paraconductivity contribution. That is the reason which this contribution is omitted near the transition.

iii) Third effect have a purely quantic nature and consist of fluctuations generated by elastic scattering of coherent 
electrons, which conform the Cooper pairs. This is known as anomalous contribution of Maki-Thompson (1989), which some time is important on the conductivity near $T_{C}$. This contribution is extremely sensible to processes that modify the electronic wave function. So, inelastic scattering processes, as electron-phonon scattering, which origin the break of spin pairing of the electron pairs, limit the lifetime of quasi particles.

iv) Besides of these effects, in HTSC was experimentally confirmed the existence of a genuine critical regime, which is characterized by correlated fluctuations immediately above $T_{C}$. These can be described by mean of the 3D$\mathrm{XY}$ model (Pureur, Menegotto Costa, Rodrigues, Schaf, \& J. V. Kunzler, 1993; P. Pureur, R. M. Costa, P. Rodrigues Jr., J. V. Kunzler, J. Schaf, L. Ghivelder, J. A. Campá and I. Rasines, 1994). In this model, the superconducting order parameter has two components (real and imaginary) as a wave function corresponding to one condensed. This permits to infer that the thermodynamics of superconductor presents a behavior of the type 3D-XY near the transition, in analogous form to the He superfluid.

The effects of thermal fluctuations are more evident in temperatures immediately above $T_{C}$. However, some fluctuation effects are strongly relevant in $T<T_{C}$ on application of magnetic fields.

One phenomenon, which is very interesting due to diversity of possible explanation, is related to the sign reversal of the Hall response in the mixed sate of type II superconductors. This anomaly is not an exclusive characteristic of the HTSC. It was experimentally observed in conventional superconductors based on Vanadium and attributed to pinning and thermal effects (Usui, Ogaswara, Yasukochi, Tomoda, 1969). In HTSC, it was proposed that the sign inversion of the Hall resistivity is caused by granular effects (Galffy \& Zirngiebl, 1988), thermoelectric effects (Freimuth, Hohn \& Galffy, 1991), vortex dynamics (Hagen, Smith, Rajeswari, ... Lobb, 1993; Rice, Rigakis, Ginsberg \& J.M. Mochel, 1992; Ambegaokar, Halperin, Nelson \& Siggia, 1980), thermal fluctuations (Lang, Heine, Schwab, Wang, \& Bäuerle, 1994; Liu, Clinton, Smith \& C.J. Lobb, 1997), flux pinning effects (Wang, Dong \& Ting, 1994) or processes of the skew scattering type (Feigel'man, Geshkenbein, Larkin, \& Vinokur, 1995). Additionally, some HTSC exhibits a double sign reversal (Hagen, Lobb, Greene \& Eddy, 1991; Zavaritsky, Samoilov \& Yurgens, 1991; Artemenko, Gorlova \& Latyshev, 1989), introducing more difficulties on the theories to explain this anomalous phenomenon.

Section 2 is dedicated to examination of the fluctuation theory. Ginzburg-Landau theory and the feature of thermal fluctuations above and below the superconducting transition in the diagonal and Hall conductivities are remarked. Sample characteristics and experimental procedures are specified in section 3. Results of fluctuation analysis in both normal and mixed states, low and high magnetic fields, longitudinal and Hall conductivities, are discussed in section 4. At last, in section 5 the conclusions are presented.

\section{Fluctuation theory}

\subsection{Ginzburg-Landau Theory}

Ginzburg and Landau, based on the phase transitions of second order (Abrikosov, A.A., 1988), developed the phenomenological theory of superconductivity near the superconducting transition. Them proposed the existence of an order parameter, which has a null value in $T>T_{C}$ and is defined in the simplest form as a complex quantity of the type

$$
\Psi(\vec{r})=|\Psi(\vec{r})| e^{i \phi(\vec{r})},
$$

where $|\Psi(\vec{r})|^{2}=n_{s}$ represents the density of superparticles (Cooper pairs) and $\phi(\vec{r})$ is the phase.

In the conventional low temperature superconductors, the transition between normal and superconducting states is correctly described by the Ginzburg-Landau theory, which is equivalent to microscopic BSC in the limit $T \rightarrow T_{C}$ (Gor'kov, L.P., 1958; $1959 ; 1960)$. Close to $T_{C}$, in absence of magnetic field, $\Psi(\vec{r})$ is small and the density of free energy can be to expands in a power series, as performed in the phase transitions of second order, $f_{s}(|\Psi|)=f_{n}+\alpha|\Psi|^{2}+\frac{1}{2} \beta|\Psi|^{4}+\ldots$ When there is external magnetic field, it is necessary to introduce the corresponding term, $\frac{B^{2}}{8 \pi}$, in the density of free energy. Furthermore, is important to consider the energy associated to the spatial variation of $\Psi(\vec{r})$, induced by the application of magnetic fields. Then, the density of free energy is given by

$f_{s}(|\Psi|)=f_{n}(T)+\alpha|\Psi|^{2}+\frac{1}{2} \beta|\Psi|^{4}+\frac{1}{2 m^{*}}\left|\left(-i \hbar \vec{\nabla}-e^{*} \vec{A}\right) \Psi\right|^{2}+\frac{B^{2}}{8 \pi}$,

where $m^{*}$ and $e^{*}$ represents the mass and the charge of a electron pair, respectively, and $f_{n}(T)$ is the density of free energy in the normal state. Minimization of free energy (1.2) with respect to the order parameter $\Psi(\vec{r})$, and the potential vector $\vec{A}$, conduces to the fundamental equations of Ginzburg-Landau theory,

$$
\begin{gathered}
\alpha|\Psi|+\beta|\Psi|^{2} \Psi+\frac{1}{2 m^{*}}\left(-i \hbar \vec{\nabla}-e^{*} \vec{A}\right)^{2} \Psi=0, \\
\vec{j}=\frac{i \hbar e^{*}}{2 m^{*}}\left(\Psi^{*} \vec{\nabla} \Psi-\Psi \vec{\nabla} \Psi^{*}\right)-\frac{\left(e^{*}\right)^{2}}{m^{*}}|\Psi|^{2} \vec{A} .
\end{gathered}
$$

Equation (1.3) is associated to the coherence length $\xi(\mathrm{T})$, which determines the spatial response of the superconductor, while equation (1.4) is related with the London penetration 
depth, $\lambda_{\mathrm{L}}$, which determines the electromagnetic response of the superconducting material. The corresponding definitions are given by

$$
\begin{gathered}
\xi(T)=\xi(0)|\varepsilon|^{-\frac{1}{2}}, \\
\lambda_{L}=\left(\frac{\mu_{0} e^{* 2}|\Psi(\vec{r})|^{2}}{m^{*}}\right)^{-\frac{1}{2}},
\end{gathered}
$$

where $\xi(0)$ is the coherence length in $T=0$ and $\varepsilon=\left(T-T_{C}\right) / T_{C}$ is known as reduced temperature.

In $T>T_{C}$, the density of superparticles is very small. This permits the expansion of $\Psi(\vec{r})$ in a Fourier series. Introducing this series in the density of free energy (1.2) and calculating the thermal media of the density of Cooper pairs, we can be to obtain the probability of occurrence of certain value of the order parameter (in the Fourier space),

$$
w\left(\psi_{\bar{q}}\right) \propto \exp \left(-\frac{\left|\psi_{\bar{q}}\right|^{2}}{2\left\langle\left|\psi_{\bar{q}}\right|^{2}\right\rangle}\right) .
$$

Equation (1.7) shows that the probability distribution of the Fourier amplitudes, $\Psi_{\vec{q}}$, has a Gaussian characteristic. So, non-correlated thermodynamic fluctuations, obtained by mean the Ginzburg-Landau theory to $T>T_{C}$, are denominated Gaussian fluctuations. The amplitude of thermal fluctuations creases and becomes to interact, in temperatures near $T_{C}$, in the so-called genuine critical region, where the fluctuation system is dominated by a collective behavior. In this limit, Ginzburg-Landau theory is not applicable. Then, the denominated Ginzburg criterion (Varlamov \& Ausloos, 1997) is used to describe the regime, which is defined in the clean limit and in three dimensions as

$$
\varepsilon_{G}=\left(\frac{k_{B}}{8 \pi^{2}(\delta c) \xi^{3}(0)}\right)^{2}=\left|\frac{T_{G}-T_{c}}{T_{c}}\right|,
$$

where, $\delta c$ is the jump in the specific heat at $T_{C}$ and $k_{B}$ is the Boltzmann constant. This criterion defines a limit in temperature, $T_{G}$. Below $T_{G}$ the Ginzburg-Landau theory is not more valid. Then, the genuine critical interval is defined by

$$
\varepsilon \leq \varepsilon_{G}
$$

\subsection{Electric Conductivity Fluctuations at $\boldsymbol{T}>\boldsymbol{T}_{C}$}

One experimental technique, which is much utilized to study the phenomenon of thermal fluctuations near the superconducting transition, is the electric conductivity. Particularly in the normal phase, this method supplies the necessary precision to detection of diverse effects as the Aslamazov-Larkin contribution, for example. Calculus of this contribution is performed based on the microscopic theory (Aslamazov \& Larkin, 1968), but the Ginzburg-Landau theory else permits the derivation of this additional term of the conductivity. Contribution of Aslamazov-Larkin to the conductivity excess $\Delta \sigma$ depends of dimensionality of system as:

$$
\begin{aligned}
& \Delta \sigma_{A L}=\frac{e^{2}}{32 \hbar \xi(0)} \varepsilon^{-\frac{1}{2}} \quad(3 \mathrm{D}), \\
& \Delta \sigma_{A L}=\frac{e^{2}}{16 \hbar s} \varepsilon^{-1} \quad(2 \mathrm{D}), \\
& \Delta \sigma_{A L}=\frac{\pi}{16} \frac{e^{2} \xi(0)}{\hbar a} \varepsilon^{-\frac{3}{2}}
\end{aligned}
$$

where $s$ is the thickness of film in the 2D case and $a$ is the transversal section area of filament in the 1D case.

The Maki-Thompson contribution, in the 3D case, presents the same divergence of Aslamazov-Larkin 3D-paraconductivity. In the $2 \mathrm{D}$ and $1 \mathrm{D}$ cases, this contribution is not relevant for the diagonal magnetoconductivity fluctuations. However, appropriately defined, these are very significant to study of Hall conductivity fluctuation (Gor'kov, L.P., 1958; 1959; 1960). Then,

$$
\begin{aligned}
& \Delta \sigma_{M T}=\frac{e^{2}}{8 \hbar s(\varepsilon-\delta)} \ln \left(\frac{\varepsilon}{\delta}\right) \quad(2 \mathrm{D}) \\
& \Delta \sigma_{M T}=\Delta \sigma_{A L}^{1 D} \frac{4 \varepsilon}{\delta}\left[1+\left(\frac{\varepsilon}{\delta}^{-\frac{1}{2}}\right)\right]^{-1} \quad(1 \mathrm{D})
\end{aligned}
$$

where, $\delta=\left(T_{i n i}-T_{C}\right) / T_{C}$ is the pair-break parameter and $T_{i n i}$ is the temperature of superconducting transition without despairing effects.

Lawrence and Doniach (1971) develop a theory to systems that have a high planar anisotropy. They propose that in this systems the order parameters of adjacent planes are weakly coupled through Josephson junctions. In their model, the contribution of thermal fluctuations on the electric conductivity is

$$
\Delta \sigma^{L D}=\frac{e^{2}}{16 \hbar s \varepsilon}(1+2 \alpha)^{-\frac{1}{2}}
$$

where $s$ represents the superconducting interplanar distance and $\alpha=\frac{2 \xi_{c}^{2}(T)}{S^{2}}=\frac{2 \xi_{c}^{2}(0)}{S^{2} \varepsilon}$ defines the coupling parameter which models the crossover between $2 \mathrm{D}$, in high temperatures, and 3D limits near $T_{C}$. The parameter a is strongly dependent from microscopic details of system. 2D and 3D limits are quantified by the dimensionless parameter $d=\frac{S}{2 \xi_{c}(0)}$, which conduce to $\alpha=\left(2 d^{2} \varepsilon\right)^{-1}$. When $d^{2} \varepsilon>>1$ the superconducting planes are effectively uncoupled and the fluctuation regime is 
2D. In this case, equation (2.6) is similar to 2D-AslamazovLarkin equation (2.2) for systems with thickness $s$. On the contrary, when $d^{2} \varepsilon<<1$, superconducting planes are coupled and the regime is $3 \mathrm{D}$. The corresponding Lawrence-Doniach equation corresponds to Aslamazov-Larkin equation 3D (2.1). However, the anisotropic quantity $\xi(0)$ is substituted by the coherence length $\xi_{c}(0)$.

The Aslamazov-Larkin model, developed to homogeneous systems, was enhanced by Char and Kapitulnik (1988) for the case of inhomogeneous materials by mean of percolation theory. In this model, it is considered that an inhomogeneous superconductor is conformed by regions with uniform superconducting properties. The global superconductivity is conserved through the strong or weak coupling between those regions along whole material. Above $T_{C}$, the superconductor is considered homogeneous, with dimensionality $d$, for length scales of homogeneous regions $L>\xi_{p}$, where $\xi_{p}$ is the correlation of percolation. So, Aslamazov-Larkin theory predicts

$$
\Delta \sigma_{A L} \approx \varepsilon^{\frac{d}{2}-2}
$$

In the opposite case, when $L<\xi_{p}$, we can be to apply the result of equation (2.7), by considering a random fractal with spectral dimension $\tilde{d}$. Then, the Char-Kapitulnik paraconductivity is given by

$$
\Delta \sigma_{C K} \approx \varepsilon^{\frac{\widetilde{d}}{2}-2}
$$

This result is general to any fractal. In the case of percolation network, the spectral dimension of the fracton has a universal value $\widetilde{d} \approx 4 / 3$ (Alexander \& Orbach, 1982; Alexander, Laermans, Orbach \& Rosenberg, 1983).

The Ginzburg criterion defined by the equation (1.8) delimits the validity of Ginzburg-Landau theory very close to $T_{C}$. Then, the thermodynamics of superconductor is not more described by the mean field theory as in the Gaussian regimes of fluctuations. In the immediate proximity to the transition, where $T_{C}$ is different to Ginzburg-Landau critical temperature, the fluctuations interact and become to be strongly correlated. When the temperature is decreased in direction to $T_{C}$, the long range order of the correlation of fluctuations increases progressively up to turn infinite at $T=T_{C}$. Thus, the region where fluctuations become infinitely coherent is denominated genuine critical regime. The study of this region is usually effectuated by mean the theory of dynamic and static scalings (Hohenberg \& Halperin, 1977), in which the free energy is expanded in a power series of the coherent length, that is the relevant scale of longitude for the critical phenomenology near the superconducting transition. This theory predicts the occurrence of a divergence in the conductivity excess $(\Delta \sigma)$ very close to $T_{C}$, according to equation

$$
\Delta \sigma \sim \varepsilon^{-v(2+z-d+\eta)},
$$

where $v$ is the critical exponent related with the coherence length, $z$ is the dynamic critical exponent, $d$ is the dimensionality of the fluctuation system and $\eta \approx 0$ is the exponent associated with the deviation of the correlation function respect to mean field behavior. The simplest description of transition in the critical regime suggests that the properties of type II superconductors, without applied magnetic fields, are that predicted by the 3D-XY-model (Pureur, et al, 1993), in which $v \approx 2 / 3$. In his prediction, Lobb (1987) defines two regimes at the critical region. First is a static critical regime very close to $T_{C}$, with con $v \approx 2 / 3$, where the exponents $z$ and $\eta$ conserve the mean field values $(z=2$ y $\eta=0)$, resulting in a critical exponent to conductivity excess, that is given by the equation $\lambda=v(2+z+d+\eta)=2 / 3$. Second corresponds to a dynamic critical regime, closer to $T_{C}$, where the effects of dynamic scaling are relevant. Then, occurs a change of the dynamic critical exponent, which acquire the value $z=$ $3 / 2$. In this case, it is predicted that the critical exponent of conductivity excess in 3D systems is given by the expression $\lambda=v(2+z+d+\eta)=1 / 3$.

\subsection{Behavior of Electric Conductivity at $T<T_{C}$}

One very interesting aspect in the granular superconductors is the occurrence of a two-step process in the normalsuperconductor transition (Pureur, et al, 1993; Gerber, Grenet, Cyrot \& Beille, 1990). This phenomenon is described by supposing that the electronic pairing stabilizes a superconductor state in mesoscopic regions (grains) of the sample, very close to the bulk $T_{C}$. On lower temperatures, another critical temperature, $T_{C O}$, conduce the system to a state with effective long range order of the phase coherence, by mean like percolative processes which active the weak junctions between diverse grains. At $T<T_{C O}$, is reached the rigorously null resistivity-state (Jurelo, Abrego Castillo, Roa-Rojas, Ferreira, Ghivelder, Pureur, Rodrigues, 1999).

The theoretical description of this two-step transition is performed in terms of a paracoherent-coherent phase transition, which is proposed to granular superconductors (Rosenblatt, Raboutou, Peyral \& Lebeau, 1990; RoaRojas, Menegotto Costa, Pureur \& Prieto, 2000). In this transition, the fluctuant phases of the Ginzburg-Landau order parameter into the grains acquire long range order. One scheme of this proposal is shown in figure 1 .

As in the vortex-glass model (Rosenblatt, et al, 1990; RoaRojas, et al, 2000), the phenomenology is described through the tunneling Hamiltonian of Cooper pairs given by

$$
H=-\sum_{\langle i, j\rangle} J_{i j} \cos \left(\theta_{i}-\theta_{j}-A_{i j}\right),
$$

where, $J_{i j}$ is the intergranular coupling energy and $q_{i}, q_{j}$ are the phases $i$ and $j$ of the order parameter, respectively. Frustration is introduced by the phase factor $A_{i j}=\frac{2 \pi}{\Phi_{0}} \int_{i}^{j} \vec{A} . d \vec{l}$, 
where $\vec{A}$ is the potential vector and the line integral is valued from center of grain $i$ to center of grain $j$. In absence of magnetic field, Hamiltonian is formally the same of a disordered 3D-XY-ferromagnet (Jurelo, et al, 1999). When magnetic field are applied, the frustration factor $A_{i j}$ conduce the system to a like spin-glass state (Rosenblatt, et al, 1990; Roa-Rojas, et al, 2000).

Then, near to $T_{C 0}$ a critical region takes place which extension can be to estimate by mean of the renormalized Ginzburg criterion (Jurelo, et al, 1999). Resistivity measurements permit to identify the paracoherent and coherent regions as observed in figure 2. It is important to remark that the resistivity in the paracoherent regime is related to activation and inactivation processes of the weak junctions into material.

Model represented by the intergranular tunneling Hamiltonian belongs to the universality class $3 \mathrm{D}-\mathrm{XY}$. This implies the occurrence of a phase transition paracoherent-coherent of second order at the temperature value $T_{C O}$, where the phase of the order parameter turns identical to all grains of material. Strong evidences of existence of this transition were reported in studies of conductivity excess and specific heat measurements. ${ }^{[25]}$

\subsection{Magnetic Effects on Conductivity at $\boldsymbol{T}>\boldsymbol{T}_{C}$}

When applied magnetic field is increased, the occupied volume by the fluctuations decrease up to turns minor that the coherence length $\xi(T)$ (Tinkham, M., 1975). On the other hand, in sufficiently strong magnetic fields, the quasiparticles are effectively confined in the lowest Landau level (LLL), due to quantization of the electronic states round the axe of application of magnetic field (Bergmann, G. 1969). Is denominated LLL the state where transversal fluctuations of magnetic field are suppressed due to separation of Landau

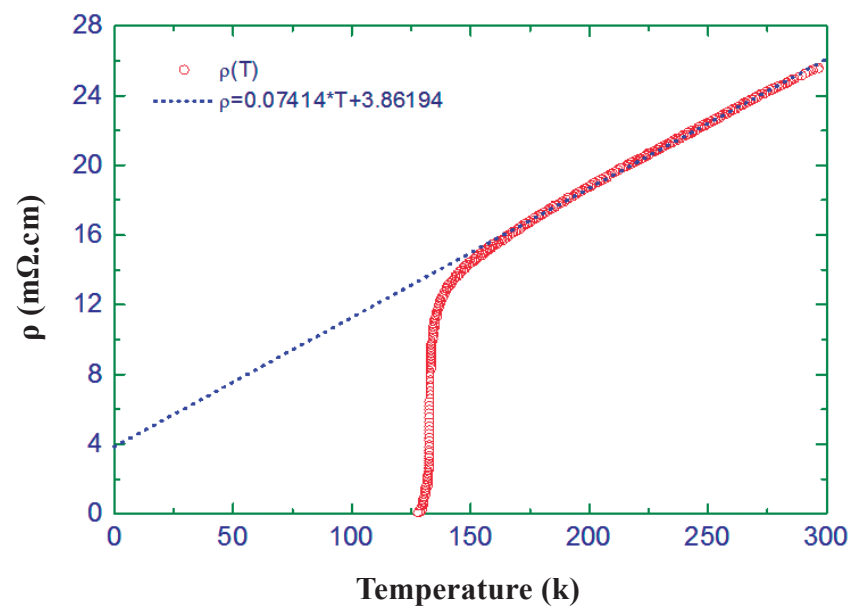

Figure 1. Linear behavior of resistivity at high temperatures and extrapolation to obtain the residual contribution at $\mathrm{T}=0 \mathrm{~K}$. orbital and are characterized by a length scale determined by the magnetic field. At sufficiently strong magnetic fields, this length scale is given by $l_{H}=\left(\frac{\Phi_{0}}{2 \pi H}\right)($ Kim $\&$ Trochet, 1992). In these circumstances, the dimensionality of system is reduced and the fluctuations acquire an effectively one-dimensional character along of magnetic field orientation. When magnetic field are applied parallel to crystallographic axe $c$ in a thin film, the characteristic volume of a typical fluctuation is $l_{H}^{2} s$, where $s$ corresponds to the thin film thickness (Gerber, et al, 1990). This reduction on effective dimensionality increases the relevance of fluctuations in certain region, near $T_{C}(H)$, which creases proportionally to field creasing, according to the Ginzburg criterion as a function of magnetic field (Ikeda, Ohmi, Tsuneto, 1989).

$$
G(H)=\left(\frac{8 \pi \kappa^{2} k_{B} T_{c} H}{\Phi_{0} \xi_{c} H_{c 2}^{2}}\right)^{\frac{2}{3}},
$$

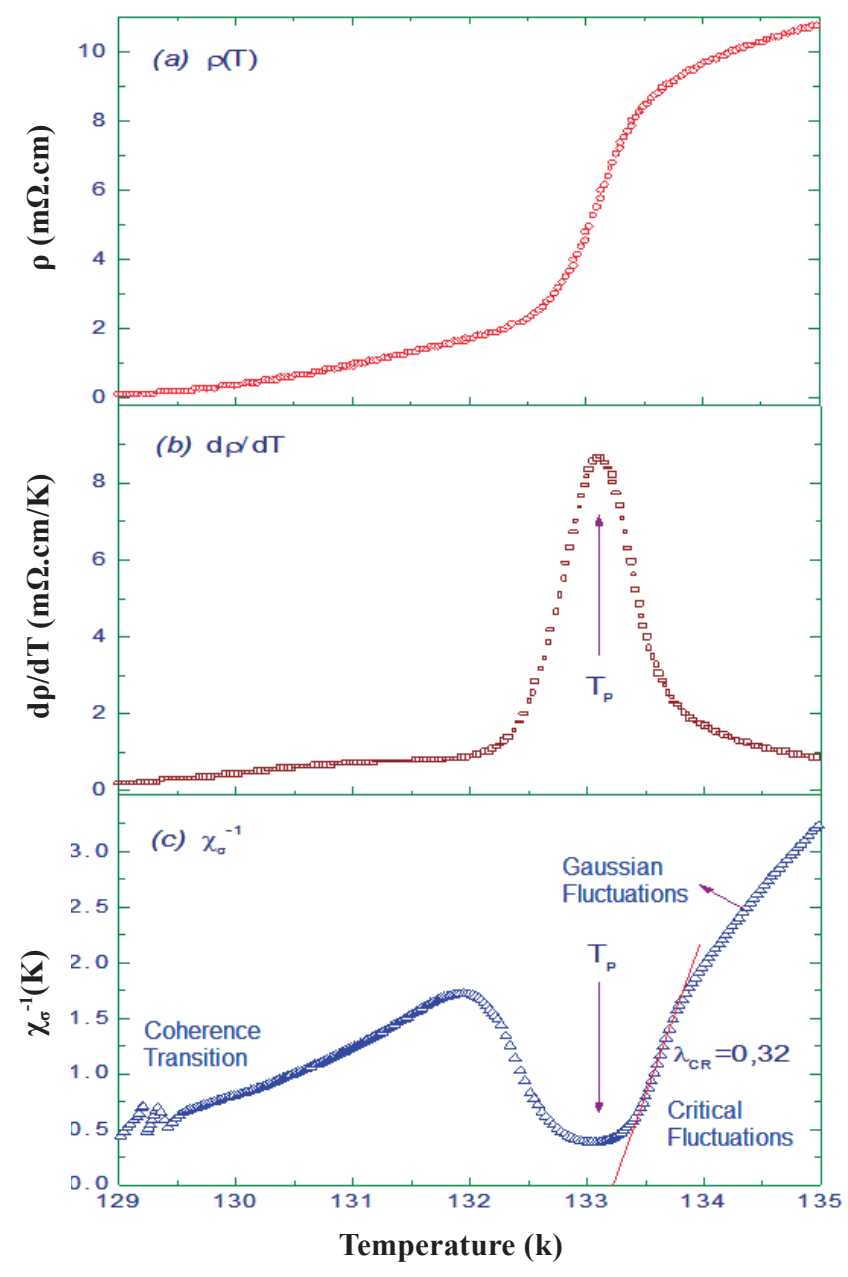

Figure 2. Characteristics of the (a) resistivity transition, (b) temperature derivative of resistivity and (c) inverse of logarithmic derivative of the conductivity excess for the $\mathrm{Hg}(\mathrm{Re})-1223$ sample. 
where $\kappa=\frac{\lambda}{\xi}$ is the Ginzburg-Landau parameter and $H_{c}$ represents the thermodynamic critical field.

On the practice, the critical behavior of the type 3D-XY is applicable to a few interval of magnetic fields, in the proximity of $H=0$. This means that is possible to observe experimentally the genuine critical regime, still in presence of weak magnetic fields. In a 3D system, the conductivity excess follows a scale law (Salamon, Shi, Overend \& Howson, 1993)

$$
\Delta \sigma H^{\frac{1}{2}}=f\left(\frac{\varepsilon}{H^{\frac{1}{2 v}}}\right),
$$

which relates the critical fluctuations with a behavior dominated by the static critical regime 3D-XY. Then, the corresponding dynamic critical exponent is $v=2 / 3$. Very close to $T_{C}$, the dynamic critical region is experimentally accessible at certain values of applied magnetic field. In high magnetic fields, occur a progressive suppression of critical and Gaussian regimes and the fluctuation system turns effectively $1 \mathrm{D}$, i.e., is confined into the lowest Landau level (Bardeen \& Stephen, 1965). In this limit case and to 3D systems, a power law governs the conductivity excess:

$$
\Delta \sigma=\left(\frac{T^{2}}{H}\right)^{1 / 3} F_{3 D}\left[A \frac{T-T_{c}(H)}{(H T)^{2 / 3}}\right] .
$$

\subsection{Magnetic Effects on Conductivity at $\boldsymbol{T}<\boldsymbol{T}_{C}$}

Presence of magnetic fields affects meaningfully the superconducting transition at $T<T_{C}$, as shown in figure 3. Picture $3 \mathrm{a}$ reveals that in low magnetic fields the intergranular regime of the superconducting transition is affected, while figure $3 \mathrm{~b}$ shows that high magnetic fields become to influence the intragranular region. Various models have been proposed to explain the behavior of HTSC in the mixed phase. More relevant are the classic of flux flow and flux creep (Bardeen \& Stephen, 1965; Anderson \& Kim, 1964), superconducting glass (Ebner \& Stroud, 1985; Rodrigues, Schaf \& Pureur, 1994; Morgenstern, Müller \& Bednorz, 1988) and vortex glass (Roa-Rojas, J., 2002; Fisher, Fisher \& Huse, 1991). Last is based on dynamic scaling theory, as far as results particularly interesting to describe the characteristic fluctuations in the mixed phase.

Vortex glass model considers that the flux lines, typical of the mixed state, adopt the configuration of magnetic ordering which occurs in the spin glasses, where the atomic magnetic moments are fix on time but are not oriented on magnetic field direction, as in ferromagnetic or antiferromagnetic materials. Spin glasses are magnetically disordered and frustrated. Disorder remarks the importance of establishing of a global state on system in which the interactions between all spin pairs can be simultaneously satisfied. Consequently, the fundamental state of a spin glass is highly degenerated, consisting of most not equivalent configurations. By this analogy, the disordered solid phase in HTSC is denominated vortex glass phase (Roa-Rojas, J., 2002; Fisher, et al, 1991) and is characterized by magnetic frustration and disorder, due to existence of pinning centers which immobilize the vortex lines.

In the limit case of like granular disorder, the vortex glass phase can be formally studied through the Hamiltonian given by equation (2.10), $\mathrm{H}=-\sum_{\langle i, j\rangle} J_{i j} \cos \left(\theta_{i}-\theta_{j}-A_{i j}\right)$. In this theory, the phenomenon of dissipation is analyzed in terms of the phase correlation of the order parameter. On the vortex glass state, where the phase of the order parameter is correlated, the longitudinal resistivity is strictly zero, originating a true superconducting state. Vortex glass transition, which occurs for a certain value of temperature $T_{g}$, is continuous and has place between the vortex-liquid and vortex-glass phases, as observed in figure 4. Most authors relate this fusion line with the phenomenon known as irreversibility line (Yeshurun \& Malozemoff, 1988; Houghton, Pelkovits \& Sudbf, 1989; Matsushita, T., 1993).

Some divergent quantities and universal laws of scaling characterize the system in the vortex glass transition. Particularly, the relevant length for this case is $\xi_{\text {, }}$, which represents the magnitude scale of correlation of the phase of the order parameter. By this reason, $\xi_{g}$ is known as coherence length of the vortex glass phase. The divergence in $T_{g}$ is given by (Koch, Foglietti \& Gallagher, 1989; Koch, Foglietti \& Fisher, 1990)

$$
\xi_{g} \propto\left(T-T_{g}\right)^{-v},
$$

where $v$ is the critical exponent related to $\xi_{g}$. Associated to this correlation length there is a relaxation time, whose scaling law can be written as $\tau=\xi_{g}^{z}$, where $z$ is the dynamic critical exponent. Transport properties near $T_{g}$ are discussed in terms of scaling laws. When a test current density is applied to system on a length $L_{s c} \approx c k_{B} T / \Phi_{0} j^{d-1}$, the regimes above $T_{g}$ can be analyze as (Abrikosov, A.A., 1988):

i) In low applied current and $L_{s c}>\xi_{g}$, the phases of the order parameter are not correlated due to thermal fluctuations. It is expected that the electric response have an ohmic behavior. When temperature is reduced in direction to $T_{g}$, the coherence length of the vortex glass $\xi_{g}$ creases while the resistance diminishes up to the zero resistance state, following a power law dependence in temperature

$$
\rho(T)=A\left(T-T_{g}\right)^{s},
$$

where $s=v(z+2-d)$ and $d$ represents the dimensionality of system. The characteristic value of $s$ in the $\mathrm{RBa}_{2} \mathrm{Cu}_{3} \mathrm{O}_{7-\delta}$ is 4 (approximately) (Roa-Rojas, et al, 2000). 
ii) When $L_{s c}<\xi_{g}$, the applied current breaks the phase correlation of the order parameter, originating a dynamic of vortex lines. From the dynamic scaling is expected a non-ohmic behavior, in the power law form, on the $I-V$ characteristic.

\section{Experimental procedures}

The synthesis of a polycrystalline $\mathrm{Hg}_{0.82} \mathrm{Re}_{0.18} \mathrm{Ba}_{2} \mathrm{Ca}_{2} \mathrm{Cu}_{3} \mathrm{O}_{8+\delta}$ superconductor was performed by means of the sealed quartz tube technique. Rhenium was added to the multiphasic precursor as $\mathrm{ReO}_{2}$ within a stoichiometric ratio. The resulting material was blended with $\mathrm{HgO}$ in order to form the final compound, following a procedure which is described in reference 49 and references therein. The obtained pellet was partially powdered for x-ray diffraction experiments. The obtained cell parameters from Rietveld analysis of the data are $a=3.8519(6) \AA$ and $c=15.686(3) \AA$. Small amounts of $\mathrm{BaCuO}_{2+\delta}$ were detected. The often present residual phases $\mathrm{HgCaO}_{2}$ and $\mathrm{Hg}(\mathrm{Re})-1212$ could be eliminated by a proper choice of the partial pressure of oxygen in the cell (Sin, Cunha, Calleja, ... Obradors, 1999). Samples prepared with the same procedure were further characterized by energydispersive $\mathrm{X}$-ray analysis (EDAX). A bar-shaped sample with dimensions $8 \times 4 \times 0.34 \mathrm{~mm}^{3}$ was cut out from the $\mathrm{Hg}(\mathrm{Re})-1223$ pellet for transport measurements. Six contacts were attached to the sample with silver paint in the conventional arrangement for simultaneous measurements of the longitudinal and Hall voltages. A low-frequency alternating current (ac) technique, which employs a lock-in amplifier as a null detector, was used to measure the transport voltages. In the case of the Hall-effect experiments, the longitudinal voltage was used as the primary source for the compensation signal in order to eliminate any spurious effect from magnetoresistance. The details of this technique were reported by Friederich (1976). The current density was fixed at $1.45 \mathrm{~A} . \mathrm{cm}^{-2}$ in all transport measurements. Temperatures were determined with a carbon-glass sensor and a Pt resistor corrected for magnetoresistance effects. Fields varying from 0 to $5 \mathrm{~T}$ were produced with a superconductor solenoid. The accuracy and the large number of recorded data points allowed us to calculate the temperature derivative of the longitudinal resistivity in the region of the superconducting transition. Structural characterization of these $\mathrm{Hg}(\mathrm{Re})-1223$ samples was extensively studied by M.T.D. Orlando et al (Sin, Cunha, Calleja, ... Obradors, 1998).

The determination of the irreversibility line was obtained from magnetoresistivity measurements and conventional magnetization experiments. First were performed in the magnetoresistometer defined above and second by means a commercial SQUID magnetometer. The zero field cooling (ZFC) and field cooled cooling (FCC) prescriptions were performed to the determination of the irreversibility line in both experimental techniques (Roa-Rojas, et al, 2000; Friederich, A., 1976).

\section{Results and discussion}

\subsection{Analysis Method and Resistive Transition}

The analysis of results for the fluctuation contribution on magnetoconductivity is performed by assuming that the conductivity excess is given by (Pureur, et al, 1993)

$$
\Delta \sigma=\sigma-\sigma_{R},
$$

where $\sigma=\sigma(T, B)$ is the measured magnetoconductivity, i.e., $\sigma(T, B)=1 / \rho(T, B)$, with applied field $B$, and $\sigma_{R}=1 / \rho_{R}$ is the regular term extrapolated from the high-temperature behavior, as shown for several samples in figure 1. Notice that the feature of the normal resistivity as a function of temperature is approximately linear, which permits to perform an easy linear extrapolation to determine $\rho_{R}$. According to the Aslamazov-Larkin proposal, the fluctuation magnetoconductivity diverges as a power law of the type

$$
\Delta \sigma(T, B)=A \varepsilon^{-\lambda}
$$

where $A$ is a constant, $\varepsilon=\frac{T-T_{c}(B)}{T_{c}(B)}$ is the field-dependent reduced temperature and $\lambda$ is the critical exponent.

Analogously to the Kouvel-Fisher method of analysis of critical phenomena (Roa-Rojas, Pureur, Orlando, BaggioSaitovitch, 2000), the logarithmic temperature derivative of $\Delta \sigma$ is given by $\frac{d}{d T} \ln (\Delta \sigma)$. Then, is defined the inverse of the logarithmic temperature derivative as the quantity

$$
\chi_{\sigma}=-\frac{d}{d T} \ln (\Delta \sigma)=\frac{1}{\Delta \sigma} \frac{d(\Delta \sigma)}{d T} .
$$

By substituting equation (4.2) in equation (4.3) it is obtained that

$$
\frac{1}{\chi_{\sigma}}=\frac{1}{\lambda}\left[T-T_{c}(B)\right]
$$

Thus, obviating more complex procedures of adjustment, simple identification of linear temperature behavior in plots of $\frac{1}{\chi_{\sigma}}$ vs $T$ allows simultaneous determination of critical temperature $T_{C}$ of fluctuation regime and the corresponding critical exponent, $\lambda$. At $T<T_{C}$, by using the same analysis method, we denote the critical exponents related with the paracoherent-coherent transition as $\lambda_{\mathrm{p}}$.

The main source of uncertainty in the data analysis comes from the extrapolation procedure to estimate $\sigma_{R}$ near $T_{C}$ and from the numerical procedure to determination of the temperature derivative of conductivity excess

$$
\frac{d(\Delta \sigma)}{d T}=-\frac{1}{\rho^{2}} \frac{d \rho}{d T}+\frac{1}{\rho_{R}^{2}} \frac{d \rho_{R}}{d T}
$$

and the logarithmic derivative of Ds

$$
\chi_{\sigma}=\left(\frac{1}{\rho^{2}} \frac{d \rho}{d T}-\frac{1}{\rho_{R}^{2}} \frac{d \rho_{R}}{d T}\right) /\left(\frac{1}{\rho}-\frac{1}{\rho_{R}}\right) .
$$


Errors introduced by the numerical calculation of equation (4.5) and (4.6) are partially compensated because the term involving $\rho_{R}$ is small compared to the term containing the total resistivity $\rho(T, B)$ near $T_{C}$. Figure 2 exemplifies the graphic analysis method by means of adjust of equation (4.4) for the $\operatorname{Hg}(\operatorname{Re})-1223$ sample. Picture (2a) shows the resistive transition $\rho(T, B=0)$, (2b) exhibits the temperature derivative $\frac{d \rho(T, B=0)}{d T}$ and (2c) presents the corresponding logarithmic derivative of the conductivity excess $\frac{1}{\chi_{\sigma}(T, B=0)}$ as a function of temperature. In (2c) it is possible to determine the critical exponents and the respective critical temperatures of the fluctuation regimes, by means of successive straight lines which can be fitted to limited but reproducible temperature ranges corresponding to these regimes.

By utilizing the temperature derivative of resistivity as a function of the temperature, the bulk critical temperature $T_{p}$ for the examined samples were obtained by assuming that the temperature position $T_{p}$ of the sharp maximum in $\frac{d \rho}{d T}$ corresponds approximately to the bulk critical temperature. As a results of resistivity measurements, width of the superconducting transition was $\Delta T_{C}=T_{p}-T_{C 0}=4.2 \mathrm{~K}$, with $T_{p}=133.2$ $K$ and $T_{C 0}=129.0 \mathrm{~K}$.

\subsection{Magnetoconductivity Fluctuations at $\boldsymbol{T}>\boldsymbol{T}_{C}$}

In the normal state, at temperatures sufficiently far away from $T_{C}$, effects of Gaussian fluctuations predominate in the electrical conductivity, as exemplified in figure 3. From the analysis of experimental data, four regimes of power law dominate by Gaussian fluctuations were identified through the exponents $\lambda_{G 3}, \lambda_{G 2}, \lambda_{G 2-G 1}$ and $\lambda_{G 1}$, as showed in table 4.1 . Meanwhile, note that $\lambda_{G 3}$ was observed only for $H<0.5 \mathrm{kOe}$.

The analysis of results was performed based on the Azlamazov-Larkin theory (1968) for fluctuations in the electrical conductivity. According with this theory the exponents are given by

$$
\lambda=2-\frac{d}{2},
$$

where $d$ is the dimension of the fluctuation space. Then, this region identified by $\lambda_{G 3}=0.53( \pm 0.02)$ corresponds to a homogeneous 3D Gaussian regime. With increasing temperature, the exponent $\lambda_{G 2}=1.02( \pm 0.04)$ corresponds to a dimensionality $d=2$ and, therefore, to $2 \mathrm{D}$ homogeneous regime. The farthest region from $T_{C}$, identified by $\lambda_{G I}=$ $1.52( \pm 0.04)$, corresponds to a $1 \mathrm{D}$ filamentar homogeneous regime. The intermediate region between $2 \mathrm{D}$ and $1 \mathrm{D}$, defined by $\lambda_{G 2-G I}=1.32( \pm 0.04)$, corresponds to a inhomogeneous fluctuational regime with spectral dimension given by the Char-Kapitulnik model as $\widetilde{d}=1.35$. This dimensionality is very close to fractal dimensiono $\mathrm{f}$ the site-percolation problem $\widetilde{d}=4 / 3$ (Char, K. \& Kapitulnik, A., 1988).

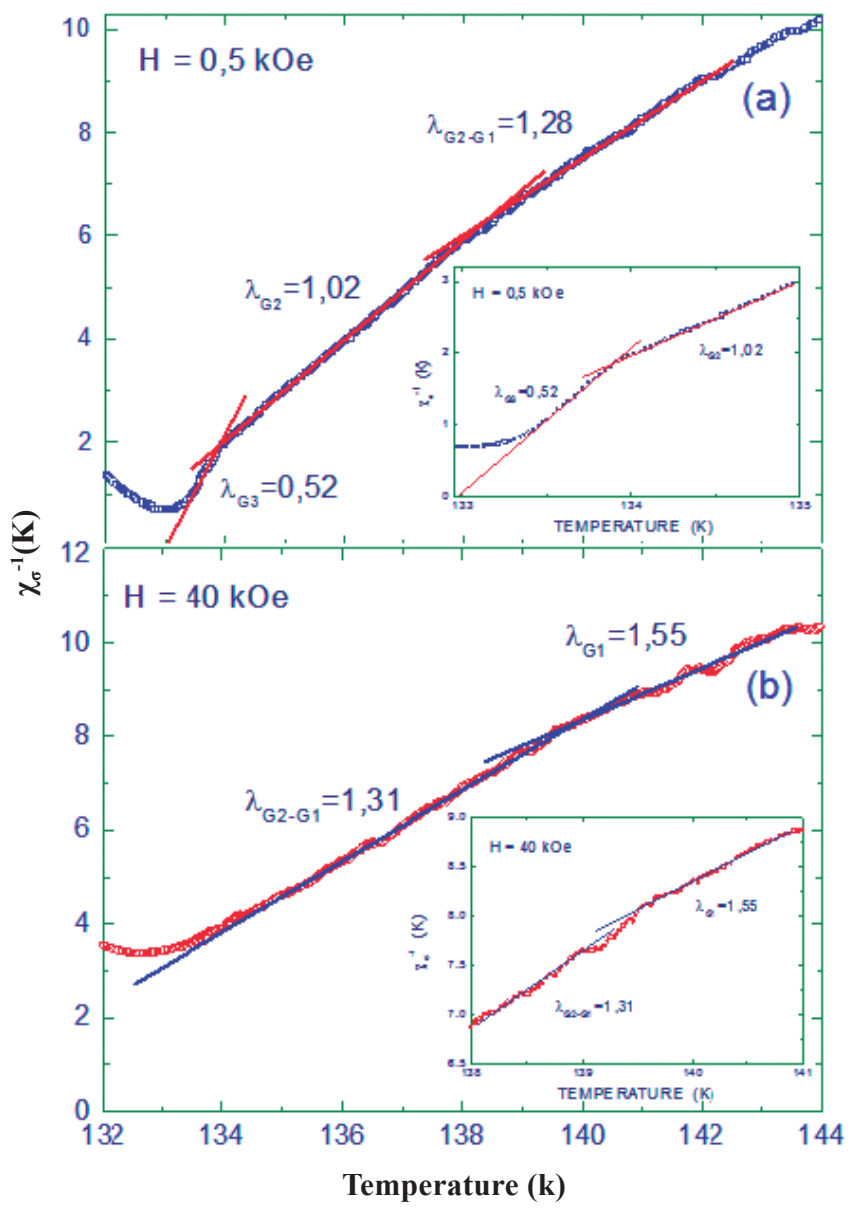

Figure 3. Gaussian fluctuation regimes identified for the $\mathrm{Hg}(\mathrm{Re})$ 1223 sample.

Table 4.1: Gaussian exponents for the $\mathrm{Hg}(\mathrm{Re})-1223$ sample. Values of $\mathrm{T}_{\mathrm{p}}$ correspond to the maximum in $\mathrm{d} \rho / \mathrm{dT}$.

\begin{tabular}{|c|c|c|c|c|c|}
\hline \multirow{4}{*}{$\underset{(\boldsymbol{k O e})}{\boldsymbol{H}}$} & \multirow{4}{*}{$\begin{array}{c}T_{P} \\
(\boldsymbol{K})\end{array}$} & \multicolumn{4}{|c|}{ Gaussian Exponents } \\
\hline & & $\lambda_{G 3}$ & $\lambda_{G 2}$ & $\lambda_{G 2-G 1}$ & $\lambda_{G 1}$ \\
\hline & & $133.7<T<134.1$ & $134.1<T<134.8$ & $134.8<T<142.2$ & $138.5<T<146.1$ \\
\hline & & $0.0044<\varepsilon<0.007$ & $0.010<\varepsilon<0.016$ & $0.015<\varepsilon<0.073$ & $0.05<\varepsilon<0.10$ \\
\hline 0 & 133.13 & $0.54 \pm 0.03$ & $1.06 \pm 0.03$ & & \\
\hline 0.5 & 133.07 & $0.52 \pm 0.02$ & $1.02 \pm 0.03$ & $1.28 \pm 0.04$ & \\
\hline 2.5 & 132.97 & & $0.98 \pm 0.04$ & $1.35 \pm 0.04$ & $1.46 \pm 0.04$ \\
\hline 10 & 132.73 & & $1.09 \pm 0.02$ & $1.33 \pm 0.02$ & $1.51 \pm 0.05$ \\
\hline 20 & 132.59 & & $0.94 \pm 0.05$ & $1.34 \pm 0.03$ & $1.52 \pm 0.03$ \\
\hline 40 & 132.32 & & & $1.31 \pm 0.04$ & $1.55 \pm 0.04$ \\
\hline 50 & 132.27 & & & $1.30 \pm 0.04$ & $1.58 \pm 0.05$ \\
\hline \multicolumn{2}{|c|}{ Average } & $0.53 \pm 0.02$ & $1.02 \pm 0.04$ & $1.32 \pm 0.04$ & $1.52 \pm 0.04$ \\
\hline
\end{tabular}

It is possible to estimate the correlation length of the Gaussian regimes by considering that these regimes vary as in the Ginzburg-Landau theory, according to $\xi(T)=\xi(0) \varepsilon^{-1 / 2}$. Using the coherence amplitude $\xi(0)$, typical of the Hg-based 
superconductors in the orientation parallel and perpendicular to the $\mathrm{Cu}-\mathrm{O}$ planes, $\xi_{a b}(0) \approx 20 \AA$ and $\xi_{c}(0) \approx 1 \AA$, respectively, the correlation lengths for the Gaussian regimes $\xi_{a b}(T)$ and $\xi_{c}(T)$ can be estimate (Shen, Lam \& Li, 1998).

For the 1D regime, the correlation length fall in the interval $63 \AA<\xi_{a b}(T)<72 \AA$ in the $\mathrm{Cu}-\mathrm{O}$ planes and $3.2 \AA<\xi_{c}(T)$ $<3.6 \AA$ in the $c$-axis. The result along the $c$-axis is much less than the spacing between inner layers of $\mathrm{Cu}-\mathrm{O}$ in the material structure, as shown in Figure 4 (Chmaissen, Huang, Antipov, ... Santoro, 1993). The non-homogeneous quasefilamentar regime evidences correlation length $74 \AA<$ $\xi_{a b}(T)<162 A$ and $3.7 \AA<x_{c}(T)<8.2 \AA$ for the $\mathrm{Cu}-\mathrm{O}$

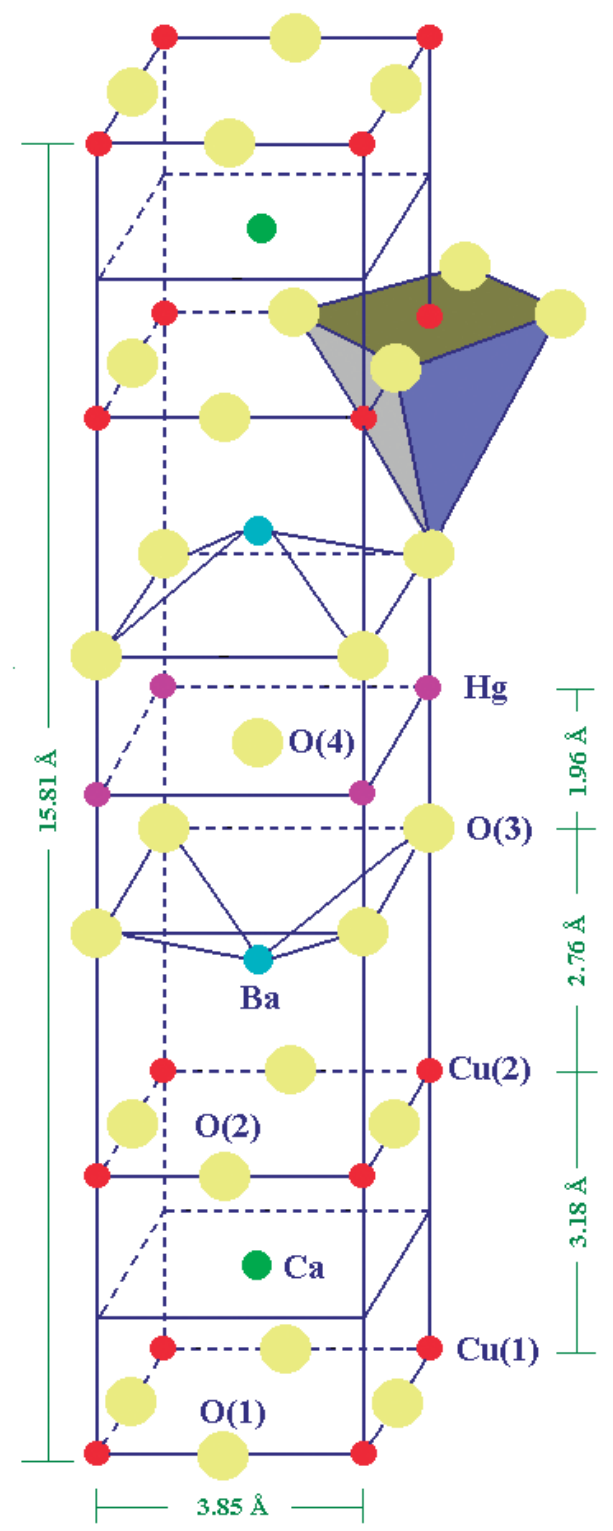

Figure 4. Crystalline structure of the Hg-1223 obtained from X-ray diffraction experiments (Chmaissen, et al, 1993). planes and in the $c$-axis respectively. The value in the $c$-axis orientation suggests that the fluctuations are restricted to the double $\mathrm{Cu}-\mathrm{O}$ planes structure. The fractality is due to the fact that the superconducting coherence length is less than the percolation correlation length, which is a consequence of disorder in the material.

For the 2D homogeneous regime, we obtained $158 \AA<\xi_{a b}(T)$ $<210 A$ and $8.2 A<\xi_{c}(T)<10.1 A$ in the $\mathrm{Cu}-\mathrm{O}$ planes and the $c$-axis respectively. The result for the $c$-axis reveals that the superconductivity is still restricted to the double $\mathrm{Cu}-\mathrm{O}$ planes of the structure.

At last, for the 3D homogeneous regime, the values of the coherence length are $239 \AA<\xi_{a b}(T)<301 \AA$ and $11.9 \AA$ $<\xi_{c}(T)<15.7 \AA$, which indicate that the superconductivity reaches $3 \mathrm{D}$-dimensional long range order.

Very close to $T_{C}$ a genuine critical regime was observed. This behavior was experimentally determined only for applied magnetic fields below $0.5 \mathrm{kO}$, which is a characteristics of the $\mathrm{RBaCuO}$ high temperature superconductors (Fabris, RoaRojas \& Pureur, 2001).

The critical exponent $\lambda_{C R}=0.32 \pm 0.01$, determined from the linear fitting in the inverse of logarithmic derivative of the conductivity excess is shown in figure 5. This exponent is explained by the 3D-XY model, according to the equation

$$
\lambda=v(2+z-d+\eta),
$$

where $v=2 / 3, \eta \approx 0, z=3 / 2$ and $d=3$, and the complex order parameter has two components, which is compatible with symmetry $s$-pure or $d$-pure of the order parameters.

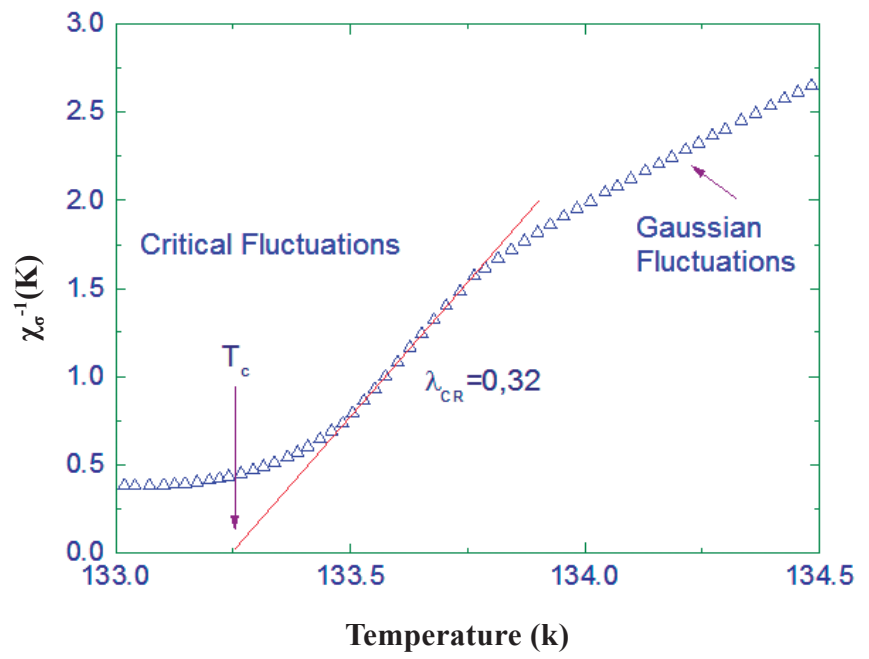

Figure 5. Genuine critical 3D-XY regime observed in the $\mathrm{Hg}(\mathrm{Re})$ 1223 sample on the application of low magnetic fields. 
A dynamical exponent $z=2$ is expected (Fisher, et al, 1991), which is characteristics of the dissipative dynamics described by the model-A of Hohenberg and Halperin (1977). However, experimental results (Roa-Rojas, Jurelo, Menegotto Costa, et al, 2000) and theoretical calculations (Lidmar, Wallin, Wengel, Girvin, Young, 1998) reveal that $z=d / 2=3 / 2$, as in the model-E of Hohenberg and Halperin, which is the dynamical universality class for the superfluid transition in ${ }^{4} \mathrm{He}$ and also for extreme type II superconductors in absence of screening (Lidmar, et al, 1998).

\subsection{Magnetoconductivity Behavior at $T<T_{C}$}

\subsection{Hall Response at $T<T_{C}$}

As reported for $\mathrm{Bi}_{2} \mathrm{Ba}_{2} \mathrm{CaCu}_{2} \mathrm{O}_{y}$ (Zavaritsky, Samoilov \& Yurgens, 1991) and $\mathrm{Tl}_{2} \mathrm{Ba}_{2} \mathrm{CaC} u_{2} \mathrm{O}_{v}$ (Hagen, Lobb, Greene \& Eddy, 1991), the Hall resistivity $\rho_{x y}$ evidenced a double signal change at temperatures below $T_{C}$ for applied fields up to $H \leq 20 \mathrm{kOe}$, as showed in figure 6 .

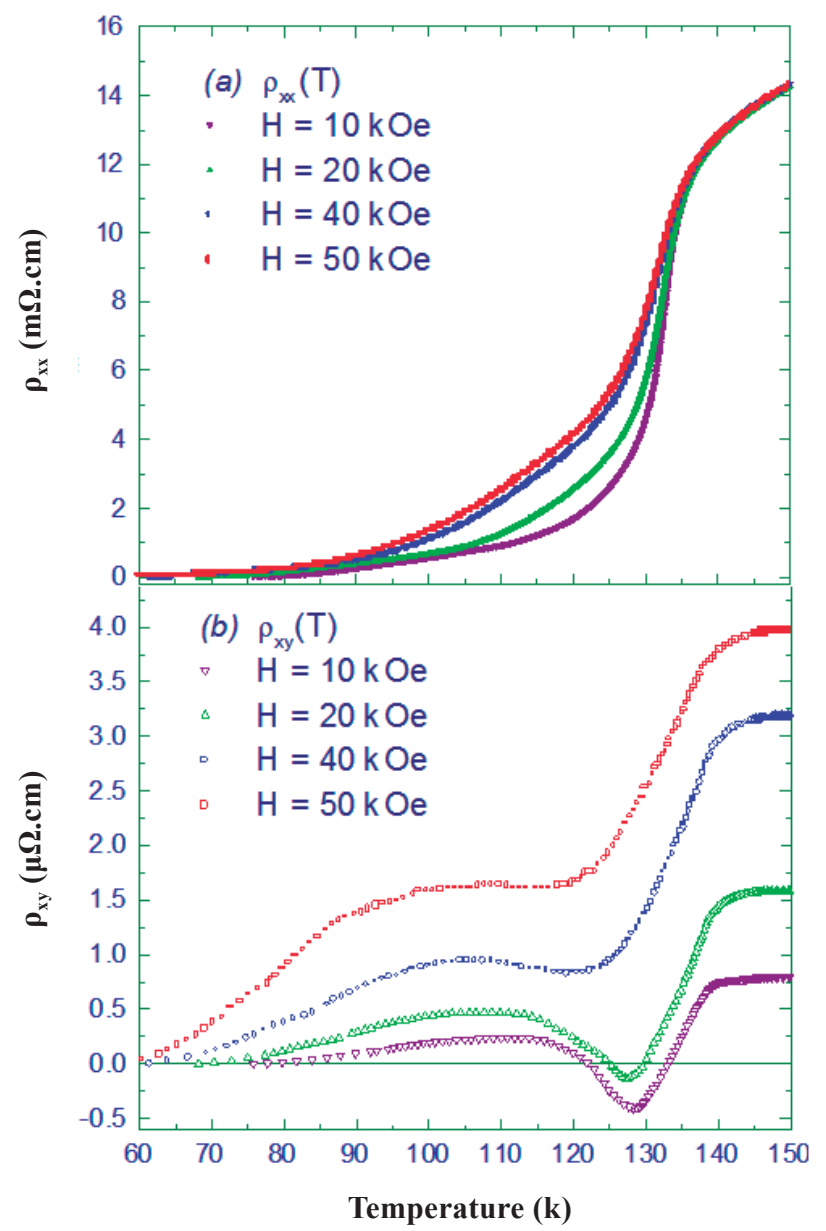

Figure 6: Behavior of the resistivity (a) diagonal $\rho_{x x}$ and (b) Hall $\rho_{\mathrm{xy}}$ on the application of magnetic fields $\mathrm{H}=10,20,40$ e $50 \mathrm{kOe}$.
On the application of high magnetic fields (above $H \leq 20$ $k O e$ ), Hall response is positive but the qualitative tendency of the curve remains as in low magnetic fields.

As showed in figure 7, close to the zero resistance state, $T_{C O}(H)$, was observed a power law of the type

$$
\left|\rho_{x y}(T)\right| \propto\left[T-T_{c 0}(H)\right]^{\beta} .
$$

The characteristic exponent determined by the scaling of figure 7 was $\beta=1.41 \pm 0.01$, which is lower that other reported for $\mathrm{DyBa}_{2} \mathrm{Cu}_{3} \mathrm{O}_{7-\delta}$ (Fabris, et al, 2001). Low values of $\beta$ are attributed to the occurrence of pinning vortex effects introduced by the planar anisotropy of this superconducting material and for granular disorder effects (Wang, et al, 1994).

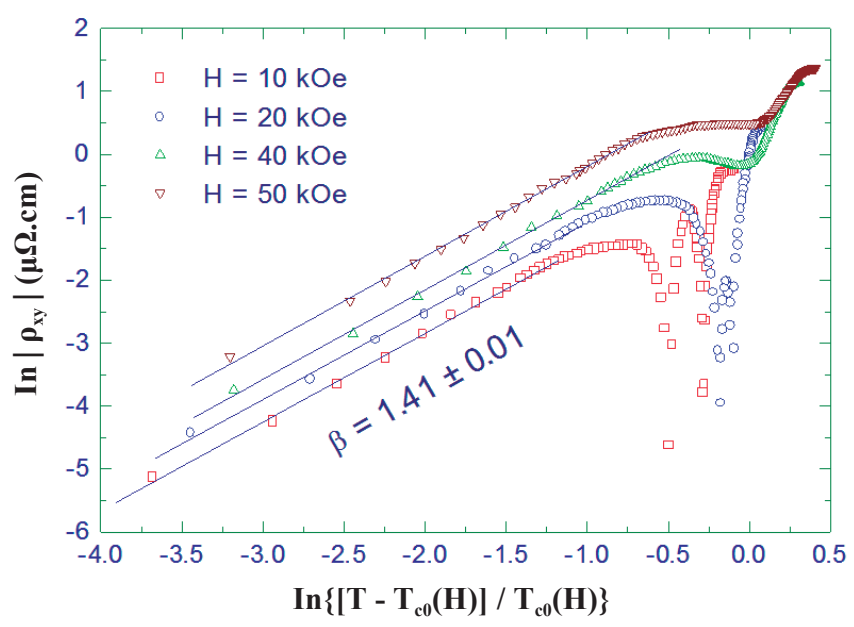

Figure 7: Scaling of the Hall resistivity at low temperatures with the equation 4.9 .

\subsection{Hall response at $\boldsymbol{T}>\boldsymbol{T}_{C}$}

In the normal state, Hall resistivity $\rho_{x y}$ varies inversely with temperature, in agreement with the figure 8 . Between $170 \mathrm{~K}$ and $260 \mathrm{~K}, \rho_{\mathrm{xy}}$ follows the behavior given by

$$
\rho_{x y}^{N}(T, H)=\frac{\mu_{0} H}{0,0084 T+0,05},
$$

where $\rho_{x y}$ is given in $\mu \Omega$.cm, $H$ in Tesla and $T$ in Kelvin.

The inverse of the Hall coefficient, $1 / R_{H}$, is given by the denominator of equation 4.10. Then, the carrier density can be schematized as showed in figure 9. The feature if carrier density in the normal state varies with temperature according to the equation

$$
n_{H}^{N}=0,62 T+3,61
$$

In units of $10^{20}$ carriers $/ \mathrm{cm}^{3}$. In figure 9 the carrier density is presented per volume of the unit cell as a function of temperature. 


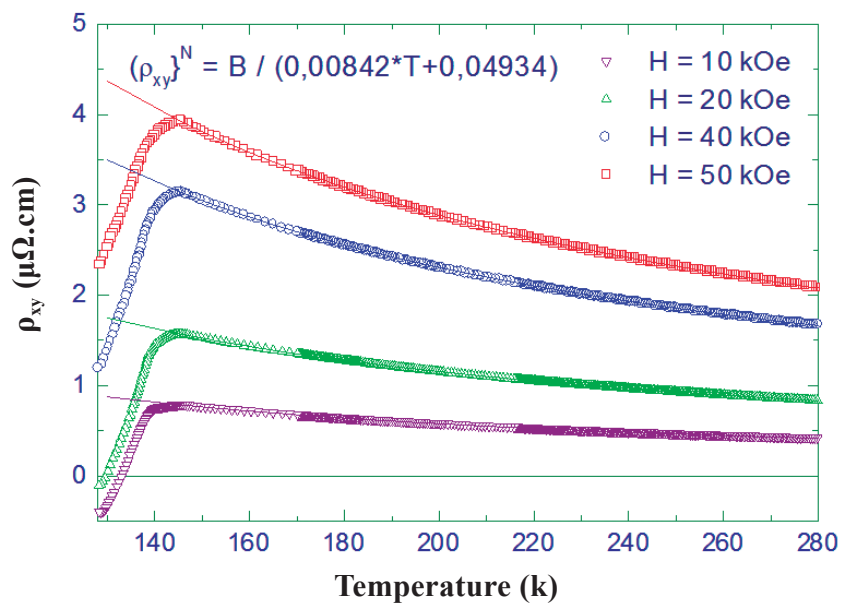

Figure 8: Normal behavior of the Hall response for $\mathrm{Hg}(\mathrm{Re})-1223$ sample.

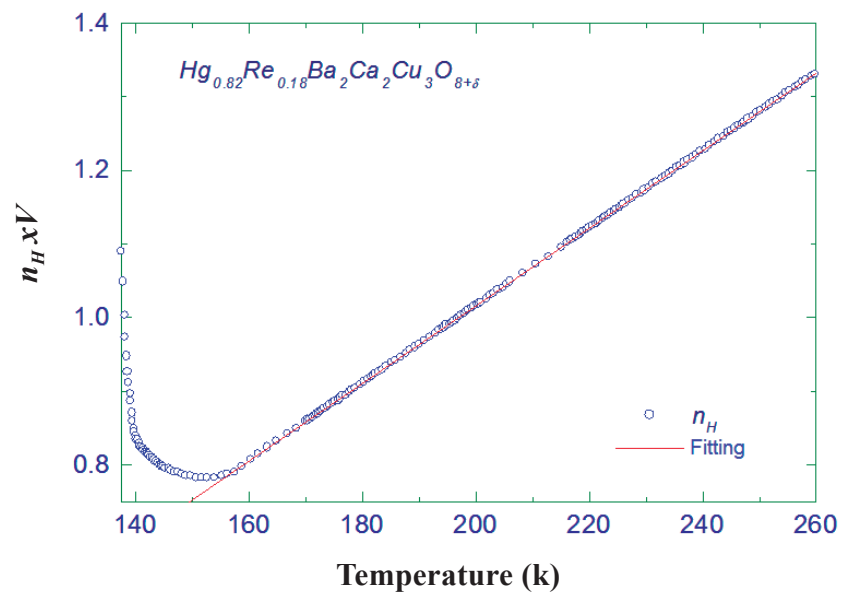

Figure 9: Carrier density in the normal state for $\operatorname{Hg}(\mathrm{Re})-1223$ material.

The Hall angle, defined as the ratio of the diagonal resistive response and the Hall resistivity (magnetoresistance), $\cot \theta_{H}^{N}=\frac{\rho_{x x}^{N}}{\rho_{x y}^{N}}$, presents a quadratic behavior with temperature, as showed in figure 10. This behavior is a universal-like behavior of the normal Hall response in high temperature superconductors (Roa-Rojas, Pureur, Mendonça-Ferreira, Orlando, Baggio-Saitovitch, 2001), according with the Anderson's formula of equation 4.12 (Anderson P. W., 1991)

$$
\operatorname{Cot} \theta_{H}=\alpha T^{2}+\beta \text {. }
$$

\section{Conclusions}

In this paper we report fluctuation magnetoconductivity analysis and Hall response in polycrystalline samples of $\mathrm{Hg}_{1}$. $\mathrm{Re}_{\mathrm{x}} \mathrm{Ba}_{2} \mathrm{CaCu}_{3} \mathrm{O}_{8+\delta}(\mathrm{x}=0.18)$ high temperature superconductor. Through the Kouvel-Fisher method, a genuinely critical

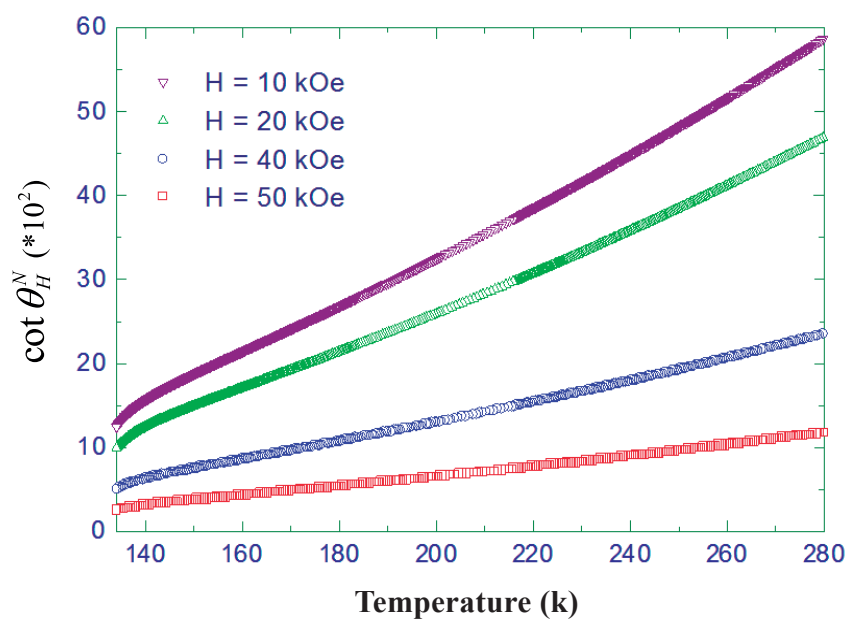

Figure 10: Hall angle for $\mathrm{Hg}(\mathrm{Re})-1223$ superconductor.

regime of fluctuations characterized by the critical exponent $\lambda_{c}=0.32 \pm 0.01$ was identified close to the critical temperature $T_{C}$ on the application of low magnetic fields. We have interpreted this result on the full dynamic 3D-XY universality class predicted by the model $\mathrm{E}$ with a dynamic critical exponent $z=3 / 2$. When the external magnetic fields $\mathrm{H} \approx 0.1 \mathrm{kOe}$, this regime becomes unstable. Above the critical temperature $T c$, four Gaussian regimes identified by the exponents $\lambda_{G 3}, \lambda_{G 2}, \lambda_{G 2-G 1}$ and $\lambda_{G 1}$ were associated to fluctuations occurring in spaces with geometry $3 \mathrm{D}$ (in the $\mathrm{Cu}-\mathrm{O}$ planes), $2 \mathrm{D}$ (when the correlation between the $\mathrm{Cu}-\mathrm{O}$ planes is weak), 2D-1D (determined by a fluctuation spectrum developing in a space with fractal topology) and 1D (corresponding to the confinement of the quasi-particles into the Lowest-LandauLevel due to the quantization of the electronic states around the axe of application of the external field). Measurements of magnetoresistance reveal the anomalous Hall response of this material. In the normal phase, the Hall resistivity is positive and varies as predicted by the Anderson's formula. In the mixed phase and for applied fields below $2 \mathrm{~T}$, the Hall resistivity shows a double sign reversal. On the application of applied fields above $2 \mathrm{~T}$, the Hall resistivity returns to the positive behavior. This behavior is interpreted as due to two independent contributions: a negative term due to thermal fluctuations which is relevant close $T_{C}$ and a positive contribution related to vortex motion which is dominant close to the zero resistance state. Below the bulk critical temperature, the Hall resistivity $\rho_{x y}$ varies as a power law of the longitudinal resistivity $\rho_{x x}$. The characteristic exponent independent of the applied magnetic field was $\beta=1.4$. This low value of the $\beta$ is attributed to vortex pinning effects introduced by the granular disorder effects which are reinforced by the planar anisotropy typical of these high temperature superconductors. 


\section{Acknowledgements}

This work was partially supported by Division of Investigations of the National University of Colombia (Hermes Code 18961) and "El Patrimonio Autónomo Fondo Nacional de Financiamiento para la Ciencia, la Tecnología y la Innovación Francisco, José de Caldas" Contract RC-No. 0850-2012.

\section{References}

Abrikosov, A.A. (1988). Fundamentals of the theory of metals. Groningen: North-Holland, p. 120-135.

Alexander, S. and Orbach, R. (1982). Density of states on fractals: "fractons". Journal Physique Letters 43, L625.

Alexander, S., Laermans, C., Orbach, R. and Rosenberg, H.M. (1983). Fracton interpretation of vibrational properties of cross-linked polymers, glasses, and irradiated quartz. Physical Review B 28, 4615.

Ambegaokar, V., Halperin, B.I., Nelson, D.R. and Siggia, E.D. (1980). Dynamics of superfluid films. Physical Review B 21, 1806

Anderson P. W. (1991). Comment on "Anomalous spectral weight transfer at the superconducting transition of $\mathrm{Bi}_{2} \mathrm{Sr}_{2} \mathrm{CaCu}_{2} \mathrm{O}_{8+\delta}$ ". Physical Review Letters 67, 660 .

Anderson, P. W. and Kim, Y. B. (1964). Hard superconductivity: theory of the motion of Abrikosov flux line, Rev. Mod. Phys. 36, 39 .

Artemenko, S.N., Gorlova, I.G. and Latyshev, Y.I. (1989). Change in the sign of the Hall effect in a superconducting transition in $\mathrm{Bi}_{2} \mathrm{Sr}_{2} \mathrm{CaCu}_{2} \mathrm{O}_{x}$ single crystals. JETP Letters 49, 403.

Artemenko, S.N., Gorlova, I.G. and Latyshev, Y.I. (1989). Vortex motion and kosterlitz-thouless transition in superconducting single crystals $\mathrm{Bi}_{2} \mathrm{Sr}_{2} \mathrm{CaCu}_{2} \mathrm{O}_{x}$. Physics Letters A 138, 428.

Aslamazov, L.G. and Larkin, A.I. (1968). Effect of Fluctuations on the Properties of a Superconductor Above the Critical Temperature. Soviet Physics Solid State 10 (4), 875-880.

Bardeen, J. and Stephen, M.J. (1965). Theory of the Motion of Vortices in Superconductors. Physical Review 140, 1197.

Bergmann, G. (1969). Landau level superconducting fluctuations of type-II superconductors in a high magnetic field, Z. Phys. B 255, 430.

Char, K. and Kapitulnik, A. (1988). Fluctuation conductivity in inhomogeneous superconductors. Z. Phys. B 72, 253.

Chmaissen, O., Huang, Q., Antipov, E.V, Putilin, S.N., Marezio, M., Loureiro, S.M., Capponi, J.J., Tholence, J.L. and Santoro, A. (1993). Neutron powder diffraction study at room temperature and at $10 \mathrm{~K}$ of the crystal structure of the $133 \mathrm{~K}$ superconductor $\mathrm{HgBa} \mathrm{Ca}_{2} \mathrm{Cu}_{3} \mathrm{O}_{8+\delta}$. Physica C, 217, 265.

Di Castro, C., Castellani, C., Raimondi, N. and Varlamov, A. (1990). Superconductive fluctuations in the density of states and tunneling resistance in high-Tc superconductors. Physical Review B 42, 10211.

Ebner, C. and Stroud, D. (1985). Diamagnetic susceptibility of superconducting clusters: Spin-glass behavior. Physical Review B 31, 165.

Fabris, F.W., Roa-Rojas, J. and Pureur, P.(2001). Magnetotransport properties and the irreversibility line in ceramic $\mathrm{DyBa}{ }_{2}$ $\mathrm{Cu}_{3} \mathrm{O}_{7-\delta}$. Physica C 354, 304-308.

Feigel'man, M.V., Geshkenbein, V.B., Larkin, A.I. and Vinokur, V.M. (1995). Sign change of the flux-flow Hall effect in HTSC. JETP Letters 62, 834-840.

Fisher, D.S., Fisher, M.P.A. and Huse, D.A. (1991). Thermal fluctuations, quenched disorder, phase transitions, and transport in type-II superconductors. Physical Review B 43, 130 .

Freimuth, A., Hohn, C. and Galffy, M. (1991). Sign change of the flux-flow Hall resistance in high-Tc superconductors. Physical Review B 44, 10396.

Friederich, A. (1976). Thesis Universit'e de Paris-Sud, Centre d'Orday

Galffy, M. and Zirngiebl, E. (1988). Hall-effect of bulk YBa2 Cu3O7- $\delta$. Solid State Communications. 68, 929-933.

Gerber, A., Grenet, T., Cyrot, M. and Beille, J. (1990). Doublepeak superconducting transition in granular $\mathrm{L}-\mathrm{M}-\mathrm{Cu}-\mathrm{O}$ ( $L=P r, N d, S m, E u, D ; M=C e, T h)$ superconductors. Physical Review Letters 65, 3201.

Gor'kov, L.P. (1958). On the energy spectrum of superconductors. Soviet Physics JETP 7, 505 - 508.

Gor'kov, L.P. (1959). Microscopic derivation of the ginzburglandau equations in the theory of superconductivity, Soviet Physics JETP 9, 1364 - 1367.

Gor'kov, L.P. (1960). Theory of superconducting alloys in a strong magnetic field near the critical temperature. Soviet Physics JETP 10, 998.

Hagen, S. J., Smith, A. W., Rajeswari, M., Peng, J. L., Li, Z. Y., Greene, R. L., Mao, S. N., Xi, X. X., Bhattacharya, S., Qi Li, and Lobb, C. J. (1993). Anomalous flux-flow Hall effect: $\mathrm{Nd}{ }_{185} \mathrm{Ce}_{015} \mathrm{CuO}_{4-1}$ and evidence for vortex dynamics. Physical Review B 47, 1064.

Hagen, S.J., Lobb, C.J., Greene, R.L. and Eddy, M. (1991). Flux-flow Hall effect in superconducting $\mathrm{Tl}_{2} \mathrm{Ba}_{2} \mathrm{CaCu}_{2} \mathrm{O}_{8}$ films. Physical Review B 43, 6246.

Hohenberg, P.C. and Halperin, B.I. (1977). Theory of dynamic critical phenomena. Rev. Mod. Phys. 49, 435.

Houghton, A., Pelkovits, R.A. and Sudbf, A. (1989). Flux lattice melting in high-T superconductorsPhysical Review B 40, 6763.

Ikeda, R., Ohmi, T., Tsuneto, T. (1989). Renormalized fluctuation theory of resistive transition in high-temperature superconductors under magnetic field. Journal of the Physical Society of Japan. 58, 1377 - 1386. 
Jurelo, A.R., Castillo, I.A., Roa-Rojas, J., Ferreira, L.M., Ghivelder, L., Pureur, P, Rodrigues Jr., P. (1999). Coherence transition in granular high temperature superconductors. Physica C 311, 133.

KIM, D. H. and Trochet, M. D. (1992). Scaling behavior of fluctuation conductivity of high-temperature superconductors in a magnetic field, Physical Review B 45, 10801.

Koch, R.H., Foglietti, V. and Fisher, M.P.A. (1990). Experimental evidence for vortex-glass superconductivity in $\mathrm{YBa}-\mathrm{Cu}$ - $\mathrm{O}$. Physical Review Letters 64, 2586.

Koch, R.H., Foglietti, V. and Gallagher, W.J. (1989). Experimental evidence for vortex-glass superconductivity in $\mathrm{Y}-\mathrm{Ba}-\mathrm{Cu}-\mathrm{O}$. Physical Review Letters 63, 1511.

Lang, W., Heine, G., Schwab, P., Wang X. Z. and Bäuerle, D. (1994). Paraconductivity and excess Hall effect in epitaxial $\mathrm{YBa}_{2} \mathrm{Cu}_{3} \mathrm{O}_{7}$ films induced by superconducting fluctuations. Physical Reviev B 49, 4209.

Lawrence, W.E. and Doniach, S. (1971). KANDO, E. (Ed.) Proceedings of 12th International Conference on Low Temperature Physics. Kyoto: Academic Press of Japan, 1971. p. $361-362$

Lidmar, J., Wallin, M., Wengel, C., Girvin, S.M., Young, A.P. (1998). Critical exponents from field theory. Physical Review B 21, 3976.

Liu, W., Clinton, T.W., Smith, A.W. and Lobb, C.J. (1997). Hallconductivity sign reversal and fluctuations in $\mathrm{YBa}_{2} \mathrm{Cu}_{3} \mathrm{O}_{7-\delta}$ films. Physical Review B 55, 11802.

Lobb, C.J. (1987). Critical fluctuations in high-T, superconductors. Physical Review B 36, 3930

Maki, K. and Thompson, R.S. (1989). Fluctuation conductivity of high-T superconductors. Physical Review B 39, 2767.

Matsushita, T. (1993). On the origin of the irreversibility line in superconductors Depinning or melting of fluxoids. Physica C 214, 100.

Morgenstern, I., Müller, K. A. and Bednorz, J. G. (1988). Glass behavior of high-T superconductors. Physica C 153/155, $59-62$.

Pureur, P., Costa, R. M., Rodrigues Jr., P., Kunzler, J. V., Schaf, J., Ghivelder, L., Campá, J. A. and Rasines, I. (1994). Critical and Gaussian conductivity fluctuations in $\mathrm{YBa}_{2} \mathrm{Cu}_{3} \mathrm{O}_{7-\delta}$ and $\mathrm{Bi}_{2} \mathrm{Sr}_{2} \mathrm{CaCu} \mathrm{O}_{8}$. Physica C 235/240, 19391940.

Pureur, P., Menegotto Costa, R., Rodrigues, Jr., P., Schaf, J. and J. V. Kunzler, (1993). Critical and Gaussian conductivity fluctuations in $\mathrm{YBa}_{2} \mathrm{Cu}_{3} \mathrm{O}_{7-\delta^{\circ}}$. Physical Review B 47, 11420.

Rice, J.P., Rigakis, N., Ginsberg, D.M. and Mochel, J.M. (1992). Sign reversal of the Hall effect below $T$ in untwinned singlecrystal $\mathrm{YBa}_{2} \mathrm{Cu}_{3} \mathrm{O}_{7-\delta}$. Physical Review B 46, 11050.

Roa-Rojas, J. (2002). Evidence of two-dimensional gauge-glass behavior near the coherence transition of $\mathrm{DyBa} \mathrm{Cu}_{3} \mathrm{O}_{7-\delta}$ ultra-thin films. Modern Physics Letters B 161061.
Roa-Rojas, J., Jurelo, A.R., Menegotto Costa, R., Mendonça Ferreira, L., Pureur, P., Orlando, M.T.D., Prieto, P., Nieva, G., (2000). Fluctuation conductivity and the dynamical universality class of the superconducting transition in the high- $T$ cuprates. Physica C. 341-348, $1911-1912$

Roa-Rojas, J., Menegotto Costa, R., Pureur, P. and Prieto, P. (2000). Pairing transition, coherence transition, and the irreversibility line in granular $\mathrm{GdBa}{ }_{2} \mathrm{Cu}_{3} \mathrm{O}_{7-\delta^{\circ}}$. Physical Review B 61, 12457

Roa-Rojas, J., Pureur, P., Mendonça-Ferreira, L., Orlando, M.T.D., Baggio-Saitovitch, E., (2001). Hall effect and longitudinal conductivity in a $\mathrm{Hg}_{082} \mathrm{Re}_{018} \mathrm{Ba}_{2} \mathrm{Ca}{ }_{2} \mathrm{Cu}_{3} \mathrm{O}_{8+\delta}$ superconductor. Superconductor Science and Technology. 14, 898

Roa-Rojas, J., Pureur, P., Orlando, M.T.D., Baggio-Saitovitch, E. (2000). Hall effect in a Hg(Re)-1223 superconductor. Physica C 341-348, 1043.

Rodrigues Jr., P., Schaf, J. and Pureur, P. (1994). Field and oxygen dependence of the magnetic irreversibility line in $\mathrm{YBa}_{2} \mathrm{Cu}_{3} \mathrm{O}_{7-\delta}$. Physical Review B 49, 15292.

Rosenblatt, J., Raboutou, A., Peyral, P. and Lebeau, C. (1990). Intragranular and intergranular transitions in $\mathrm{Y}-\mathrm{Ba}-\mathrm{Cu}-\mathrm{O}$ ceramics. Rev. Physique Appl. 25, 73.

Salamon, M. B., Shi, J., Oyerend, N. and Howson, M. A. (1993). XY-like critical behavior of the thermodynamic and transport properties of $\mathrm{YBa}_{2} \mathrm{Cu}_{3} \mathrm{O}_{7-x}$ in magnetic fields near $T_{c}$. Physical Review B 47, 5520.

Shen L.J., Lam C.C., Li J.Q. (1998). Thermodynamic fluctuation under high pressure in Hg-1223 superconductors. Superconducting Science and Technology 11, 1277.

Sin, A., Cunha,A.G., Calleja,A., Orlando, M.T.D, Emmerich, F.G., Baggio-Saitovitch, E., Segarra, M., Piñol, S. and Obradors, X. (1999). Influence of precursor oxygen stoichiometry on the formation of $\mathrm{Hg}, \mathrm{Re}-1223$ superconductors. Superconductor Science and Technology 12, 120.

Sin, A., Cunha, A.G., Calleja, A., Orlando, M.T.D, Emmerich, F.G., Baggio-Saitovitch, E., Segarra, M., Piñol, S. and Obradors, X. (1998). Pressure-Controlled Synthesis of the $\mathrm{Hg}_{082} \mathrm{Re}_{018} \mathrm{Ba} \mathrm{Ca}_{2} \mathrm{Cu}_{8+\delta}$ Superconductor, Advanced Materials 10, 1126.

Stanley, H.E., (1971). Introduction to phase transitions and critical phenomena. Oxford: Clarendon Press, p.p. 180.

Tinkham, M. (1975). Introduction to superconductivity. Florida: Krieger, p.p. 230-257.

Usui, M., Ogaswara, T., Yasukochi, K., Tomoda, S. (1969). Magnetization, Flux Flow Resistance and Hall Effect in Superconducting Vanadium. Journal of the Physical Society of Japan 27, 574.

Varlamov, A.A. and Ausloos, M. (1996). Nato advanced research workshop on fluctuation phenomena in high critical 
temperature superconducting ceramics, Trieste. Edited by Ausloos, M. and Varlamov, A.A. Dordrecht: Kluwer Academic Publishers, (1997). p.p. 3-41.

Wang, Z.D., Dong, J. and Ting, C.S. (1994). Unified theory of mixed state Hall effect in type-II superconductors: Scaling behavior and sign reversal. Physical Review Letters 72, 3875 .
Yeshurun, Y. and Malozemoff, A.P. (1988). Giant Flux Creep and Irreversibility in an $\mathrm{Y}-\mathrm{Ba}-\mathrm{Cu}-\mathrm{O}$ Crystal: An Alternative to the Superconducting-Glass Model. Physical Review Letters 60, 2202.

Zavaritsky, N.V., Samoilov, A.V. and Yurgens, A.A. (1991). Transport coefficients and flux motion in $\mathrm{Bi}_{2} \mathrm{Sr}_{2} \mathrm{CaCu}_{2} \mathrm{O}_{x}$ single crystals. Physica C 180, 417. 